wiesen sich die Eiweifskörper in fraglichem Brote zu 52,62 pCt. verdaulich.

Zu ahnlichen ungtinstigen Resultaten ist auch $\mathrm{S} a \operatorname{lm} \theta \mathrm{n} \theta \mathrm{w}^{1}$ ) gelangt, welcher bei Versuchen an 2 Menschen fand, dafs Chenopodiumbrot ganz wertlos ist, sofern die stickstoffhaltigen Bestandteile zur Halfte "wertlose Nucleine" sind, und dafs vom Gesamtstickstoff nur der dritte Teil assimiliert wird. Zudem wirkt das Chenopodiumbrot brechenerregend und verletzt den Magendarmkanal.

Zum Schlusse sei nur noch kurz auf Hungerbrot anderer Länder hingewiesen, zu dessen Herstellung u. a. Stroh, Baumrinden, Knochenmehl, Sauerampfersamen u. dergl. verwendet worden sind. ${ }^{2}$ )

Von kroatischem Hungerbrot, bestehend aus Roggen, Mais und Buchenholzmehl, hat $\mathrm{J} a \mathrm{n} \theta \mathrm{c \theta k} \mathbf{k}^{3}$ ) eine ausführliche Analyse veröffentlicht, auf welche hier nur aufmerksam gemacht werden kann.

Für die meisten dieser Hungerbrotsorten ist hoher Holzfaserund Aschengehalt charakteristisch.

\title{
III. Ueber Chenopodin und den Nachweis des Chenopodiumsamens in Mahlprodukten.
}

Fon Denselben.

Nach den in der vorhergehenden Mitteilung enthaltenen Angaben ist die Annahme gerechtfertigt, dals nicht blos der hohe Holzfaserund Aschengehalt, sowio die aufsere Beschaffenheit das Chenopodiumbrot für die menschliche Ernăhrung ungeeignet macht, sondern es scheint auch, als ob der Chenopodiumsamen einen giftigen Bestandteil enthalte, dem vielleicht der bittere Geschmack des aus dem genannten Materiale hergestellten Brotes zuzuschreiben ist.

Man wird in der Annahme eines giftigen Bestandteiles im Chenopodium bestärkt, wenn man erfährt, dals die in Rede stehende

1) Ueber die chemische Zusammensetzung und Ausnutzung der Samen von Chenopodium album. Inaug. Dissert. Petersburg 1892. Aus dieser Ubermittelte uns Herr Staatsrat Prof. Dr. Kob e r t freundlichst obige Mitteilungen brieflich.

2) Konig, 3. Aufl., Bd. I, S. 640; vergl. auch : v. Bibra, Die Getreidearten und das Brot (1860).

3) Listy chem. 5. 157-159, Agram 1881, und Chomisches Centralblatt 1882, S. 266. 
Pflanze von Tieren nicht nur ungern gefressen wird, sondern dals Chenopodium rubrum $L$. Schafen direkt nachteilig werden und Chen. hybridum $L$. sich für Schweine sogar tödtlich erweisen soll. ${ }^{1}$ )

Engelhard 2) giebt vom Chen. anthelminticum an, dals es von Pferden, Rindvieh and Schafen nur im Notfalle aufgenommen wird und darmstringierend wirkt, wogegen nach $\left.\mathrm{L} \theta \mathrm{uni} \mathrm{s}^{3}\right)$ Chen. album in Ostindien als Gemüsepflanze angebaut und auch in einigen Teilen Deutschlands gesammelt und genossen wird. Auf eine Analyse von Chen. album als "Salat-Unkraut", d. h. auf ein Unkraut, welches gelegentlich als Salat gegessen wird, haben wir Eingangs unserer ersten Mitteilung aufmerksam gemacht und dort auch die bekannte Thatsache erwahnt, dafs Chenop. Quinoa als Mehlfrucht in Südamerika angebaut wird.

Die Angaben über schädliche Wirkungen von verschiedenen Chenopodien sind also widersprechend; wir haben deshalb die sich uns bietende Gelegenheit benutzt, um unseren Chenopodiumsamen einer Untersuchung namentlich auf Alkaloide zu unterziehen.

In der Litteratur findet sich nämlich eine Angabe, der zu Folge Engelhard ${ }^{1}$ ) aus Chenopodium anthelmintucum durch Extraktion mit Alkohol eine gelblich weifse Substanz von bitterlich kratzendem Geschmack, leicht löslich in Alkohol, schwer in Aether gewann. Sie war geruchlos, schmolz bei gelinder Wärme und zersetzte sich bei starkem Erhitzen unter Entwicklung von Ammoniakdämpfen. - Zu weiterer Untersuchung reichte die Substanz nicht aus, gleichwohl bezeichnete Engelhard sie als Alkaloid uud legte ihr den Namen Chenopodin bei. Denselben gab spater (1863) H. R $\theta$ in $\mathrm{sch}^{2}$ ) einer Substanz, die er aus dem ausgeprelsten Safte junger Pflanzen von Chenop. album erhalten hatte. Sie bildete ein weirses, körniges, aus mikroskopisch kleinen, kugelförmig angeordneten Nadeln bestehendes Pulver, welches bei $180-225^{\circ} \mathrm{C}$ nach vorgängiger Schmelzung und unter Entwicklung eines widrigen Geruches in weifsen krystallinischen Flocken sublimirte. Formel $\mathrm{C}_{6} \mathrm{H}_{19} \mathrm{NO}_{4}$.

1) Nobbe, Samenkunde. Berlin 1876 S. 458.

2) Archiv der Pharmazio 54292 (1848).

3) Synopsis der Naturreiche. Hannover 1885 II. Teil, Bd. 2. S. 300.

1) Dieses Archiv Bd. 54. 292 (1848).

2) Neues Jahrbuch der Pharmazio 20, 268, 21. 123, 23. 73 und 27. 193. - Husemann-Hilger, Pflanzenstoffe. 2. Aufl. Bd. I. 525. 
Dieses Chenopodin erwies sich aber später als identisch mit Leucin $\left.{ }^{1}\right)_{6} \mathrm{C}_{13} \mathrm{NO}_{2}$, welches in der jungen Chenopodiumpllanze enthalten gewesen oder bei Verarbeitung des Saftes durch Spaltung von Eiweils entstanden sein kann. R $\theta$ ins $\mathrm{c} h$ überzeugte sich dann selbst davon, dals sein Chenopodin kein charakteristisches Alkaloid, sondern Leucin war.

Den Angaben von Engelhard und Reinsch zufolge war es somit mindestens sehr zweifelhaft, ob Chenop. album uberhaupt einen alkaloidischen Bestandteil enthalte.

Zum Nachweise eines solchen verfuhren wir in bekannter Weise so, dals wir den feingemahlenen Chenopodiumsamen mehrfach mit heifsem Alkohol auszogen, letzteren von den Extrakten abdestillierten und den wässerigen Rtickstand, welcher braungefärbt war und sauer reagierte, mit einem Gemische von Aether und Petroleumäther ansschüttelten, dessen Verdunstungsrückstand keine allgemeinen Alkaloidreaktionen lieferte.

Die wässerige Flüssigkeit dagegen gab, nachdem sie durch Bleiessig gefält und nach Abscheidung des von diesem Reagens bewirkten voluminösen gelben Niederschlages durch Schwefelsäure entbleit worden war, mit Phosphorwolfram-, Phosphormolybdänsäure und Jodjodkalium Fallungen. Die durch diese Reaktionen angezeigte alkaloidische Substanz wurde nun durch Fällen mitPhosphormolybdänsäure, Zerlegung des gelben Niederschlages durch gebrannte Magnesia und Extrahieren der mit Gyps entwässerten Masse im Soxhletschen Apparate mittels Alkohols isoliert, nachdem sich berausgestellt hatte, dals Petroloumather und Äther keine Substanz aufnahm, welche auf die oben erwähnten Alkaloidreagentien wirkte.

Das alkoholische Extrakt schied beim Verdunsten einige Krystalle unorganischer Salze ab und verdickte sich schlieislich zu einem alkalisch reagierenden Sirup. Derselbe löste sich leicht in Wasser unter Abscheidung einer wachsartigen schmierigen Masse, nach deren Beseitigung die reichlich mit Alkohol vermischte wässerige Fltissigkeit beim Verdunsten uber Schwefelsäure einen eisblumenartig kristallisierten Rückstand hinterliels.

Da den Chenopodiaceen verwandte Pflanzen, wie z. B. die

1) v. Gourup-Besanez, Ber. d. Deutsch. chem. Ges. 7, 147 (1874). Drag endorff, Analyse von Pflanzen und Pflanzenteilen. Göttingen 1882. 
Runkelrübe (Beta vulgaris) Betaïn enthalten, so lag die Vermutung nahe, dals die von uns isolierte Substanz Betain sei oder doch zu diesem in naher Beziehung stehe. Wir haben sie deshalb in $\mathrm{Pa}$ rallele mit Betaïn den nachfolgenden Reaktionen unterzogen und dabei auch das $R$ eins $\mathrm{ch}^{\prime}$ 'sche Chenopodin, d. h. Leucin, in diese Prüfung mit hineingenommen. Das Resultat derselben ergibt sich aus der nachstehenden Tabelle:

\begin{tabular}{|c|c|c|c|c|c|c|c|}
\hline & $\begin{array}{l}\text { Cadmium- } \\
\text { jodidjod- } \\
\text { kalium }\end{array}$ & $\begin{array}{l}\text { Queck- } \\
\text { silber- } \\
\text { jodidjod- } \\
\text { kalium }\end{array}$ & $\begin{array}{c}\text { Wismath- } \\
\text { jodidjod- } \\
\text { kalinm }\end{array}$ & $\begin{array}{l}\text { Jodjod- } \\
\text { ralinm }\end{array}$ & $\begin{array}{l}\text { Gerb- } \\
\text { seure }\end{array}$ & $\begin{array}{l}\text { Phosphor- } \\
\text { wolfram- } \\
\text { sãare }\end{array}$ & $\begin{array}{l}\text { Phoophor- } \\
\text { molybdān- } \\
\text { sảuro }\end{array}$ \\
\hline $\begin{array}{l}\text { Substanz ans } \\
\text { Chenopodium }\end{array}$ & $\begin{array}{l}\text { keine } \\
\text { Fällang }\end{array}$ & $\begin{array}{l}\text { weifse } \\
\text { Trubuog }\end{array}$ & $\begin{array}{c}\text { brănnliche } \\
\text { amorphe } \\
\text { Fällung }\end{array}$ & $\begin{array}{l}\text { rötlich- } \\
\text { gelbe } \\
\text { Fällang }\end{array}$ & $\begin{array}{l}\text { keine } \\
\text { Fallung }\end{array}$ & $\begin{array}{c}\text { starker } \\
\text { weifser } \\
\text { Nieder- } \\
\text { schlag }\end{array}$ & $\begin{array}{c}\text { starker } \\
\text { gelber } \\
\text { Nioder- } \\
\text { schlag }\end{array}$ \\
\hline Betain & deagl. & $\begin{array}{l}\text { keine } \\
\text { Fullong }\end{array}$ & $\begin{array}{l}\text { rotgelber } \\
\text { Nieder- } \\
\text { schlag }\end{array}$ & $\begin{array}{c}\text { braunliche } \\
\text { Fallung }\end{array}$ & desgl & desgl. & desgl. \\
\hline Leoein & desgl. & desgl. & \multicolumn{3}{|c|}{ reine Fällung } & $\begin{array}{l}\text { weil'sliche } \\
\text { Trabung }\end{array}$ & $\begin{array}{c}\text { gelbliche } \\
\text { Trtibung }\end{array}$ \\
\hline
\end{tabular}

Conc. Schwefelsäure, Salpetersaure und Erdmann's Reagens riefen keine Fürbungen hervor. Vanadinschwefelsäure färbte die alkaloidische Substanz aus Chenopodiumsamen braun, Betain rotbraun und Leucin allmählich blattgrün. Fröde's Reagens zeigte keine bemerkenswerte Verănderung.

Obigen Reaktionen zufolge ist die von uns isolierte Substanz wahrscheinlich Betain, ganz bestimmt aber kein Leucin. Um weitere Anhaltspunkte zu gewinnen, haben wir unsere Substanz in ibr Platindoppelsalz ubergeführt, welches sich in federförmigen Krystallen ausschied.

Platinbestimmung: $0.1036 \mathrm{gr}$ des bei $100^{\circ} \mathrm{C}$ getrockneten Salzes hinterliefsen beim Verbrennen $0.0318 \mathrm{gr}$ Platin, entsprechend $30.69 \% \mathrm{Pt}$.

Gefunden: $30.69 \% \mathrm{Pt}$

Berechnet für $\left[\mathrm{C}_{5} \mathrm{H}_{11} \mathrm{NO}_{2}\right.$. $\mathrm{HCl}_{2} \mathrm{Pt} \mathrm{Cl}_{4}$

$$
30.38 \% \mathrm{Pt}
$$

Auf Grund dieser Zahl, sowie unter Berücksichtigung der obigen Reaktionen und der mit Betaïn ubereinstimmenden Krystallform des Platinsalzes ist die Annahme gerechtfertigt, dals die aus dem Samen 
von Chenopodium album nach einer Methode gewonnene Substanz, mittest deren man auch das Betain darstellt, thatsächlich Betain ist. Der Name Chenopodin, mit welchem Engelhard ein dem Chenopodium eigentümliches Alkaloid bezeichnen wollte, und welchen Reinsch irrtumlich dem Leucin beilegte, ist also vorläufig aus der Litteratur zu streichen.

Das Betaïn ist ein unschädlicher Stoff, der für die toxischen Wirkungen des aus Chenopodiumsamen hergestellten Hungerbrotes sicherlich nicht verantwortlich gemacht werden kann.

Auf Saponinsubstanzen und Oxalsäure, welche angeblich in einigen Chenopodiaceen vorkommen, haben wir nebenbei Rücksicht genommen, allein mit negativem Resultate. Vorläufig bleibt somit nur die Vermutung übrig, dafs das atherische Öl, von welchem wir eine ganz kleine Menge durch Destillation des Ätherextraktes mit Wasserdampf erhielten, aber nicht näher untersuchen konnten, der physiologisch wirksame Bestandteil sei, dessentwegen andere Chenopodiumarten (z. B, Chenop. anthelminticum) vorübergehend medizinisch angewendet, ${ }^{1}$ ) aber der unangenehmen Nebenwirkung wegen wieder verlassen worden sind.

Nebenbei sei erwähnt, dafs im Ätherextrakte unseres Chenopodiumsamens eine cholesterinartige Substanz enthalten war, die wahrscheinlich mit dem von Reinke und Rodewald ${ }^{2}$ ) gefundenen Paracholesterin identisch ist.

Was nun endlich den Nachweis von Chenopodiumsamen in den Mahlprodukten anbetrifft, so ist eine leicht ausführbare Methode schon aus dem Grunde erwünscht, weil das Chenopodium zu denjenigen Unkrautsamen gehört, welche man gelegentlich als verunreinigende Beimengung in Mehl und Kleie anzutreffen erwarten kann.

Von den bei bezüglichen Mehlprüfungen üblichen Orientierungsreaktionen ${ }^{3}$ ) erwies sich nur diejenige von Vogl insofern als berücksichtigenswert, als chenopodiumhaltiges Mehl bei mehrstündiger Beribhrung mit salzsaurem Weingeist, namentlich in der Wärme, eine

1) Vergl. die citirte Arbeit von Engelhard.

2) Liebig's Annalen der Chemie 207. 231.

3) Vereinbarungen der freien Vereinigung bayerischer Vertreter der angewandten Chemie. Herausgegeben von A. Hilger. Berlin 1885. S. 279 . 
blafsrosa bis tiefrote Fürbung liefert, die an sich noch kein Beweis für das Vorhandensein von Chenopodiumsamenmehl ist, die aber einen bezüglichen Hinweis futr die mikroskopische Untersuchung bietet.

\title{
Arbeiten aus dem pharmazeutischen Institut der Universität Bern. \\ 6. Untersuchungen uiber die Sekrete.
}

\author{
Mitgeteilt von $\mathbf{A}$. Ts chirch.
}

Ueber die Sekretbildung in den schizogenen Gängen.

Von A. Béch A raz.

Eingegangen am 8. September 1893.

Die Entstehung der Sekrete ist der Gegenstand zahlreicher Untersuchungen gewesen, ohne dals es bis heute gelungen ist, eine genaue Kenntnis von der Art und Weise zu erhalten, wie sich die Sekrete in den Sekretbehältern bilden. Meyen, $\left.{ }^{1}\right)$ N. J. C. Müller, ${ }^{2}$ ) Mohl ${ }^{3}$ ) und Dippel ${ }^{4}$ ) gelangen nach ihren Beobachtungen zu dem Schlusse, dafs Harz, Balsam oder Oel aus den Gewebezellen durch die Zellmembran nach den Sekretbehältern hin diffundiere.

Karsten ${ }^{5}$ ) dagegen, sowie Wigand ${ }^{6}$ ) führen die Sekretbildung in den Sekretgängen auf eine Umwandlung der Zellmembran in Harz zurück.

De Bary ") vermutet, dafs die Sekrete in den schizogenen Behältern wohl allgemein zunächst als Bestandteile der Zellwände aufzufassen seien. Eigene Beobachtungen von ihm über den Gegenstand liegen jedoch nicht vor.

Auch Hanstein ${ }^{8}$ ) und Haberlandt ${ }^{9}$ ) haben ähnliche Ansichten,

1) Meyen. Sekretionsorgane der Pflanzen. 1837.

2) N. J. C. Müller. Untersuchungen uber die Verteilung der Harze u. s. w. Pringsh. J. B. V.

3) Mohl. Ueber die Gewinnung des Terpentins. Bot. Ztg. 1859.

4) Dippel. Die Harzbehälter der Weifstanne etc. Bot. Ztg. 1863.

5) Karsten. Die Entstehung des Farzes. Bot. Ztg. 1857.

6) Wigand. Ueber die Desorganisation der Pfanzenzelle etc. Pringsh. J. B. III.

7 de Bary. Vergleichende Anatomie der Vegetationsorgane. 18i7.

8) Hanstein. Ueber die Organe der Harz- und Schleimabsonderung bei den Laubknospen. Bot. Ztg. 1868.

9) Haberlandt. Physiologische Pflanzenanatomie. 1884. 